\title{
Large amplitude wavelength modulation spectroscopy for sensitive measurements of broad absorbers
}

\author{
TORREY R. S. HAYden AND GREgory B. RIEKER \\ Precision Laser Diagnostics Laboratory, Department of Mechanical Engineering, University of \\ Colorado, Boulder, CO 80309, USA \\ *torrey.hayden@colorado.edu
}

\begin{abstract}
We demonstrate large amplitude wavelength modulation spectroscopy (WMS) with a MEMS-tunable vertical cavity surface-emitting laser (MEMS-VCSEL) to measure high-density gases. WMS enables sensitive measurements of gas phase thermodynamic properties in harsh environments, but has been limited to moderate pressure and density conditions because of the narrow tuning range of traditional DFB lasers. The MEMS-tunable laser is able to rapidly modulate across the broadened features of high-density gas mixtures to produce the harmonic signals in the detected light intensity typical of WMS. We illustrate the technique on high-pressure mixtures of $\mathrm{CO}_{2}$ in air that are 2.5 times higher density than previously published WMS measurements (equivalent to greater than 255 atm at $1500 \mathrm{~K}$ ). We develop a WMS model that accounts for nonlinear tuning of the laser to enable extraction of thermodynamic properties from measured data. The agreement of the measured data and model suggests that this technique could be used now for calibrated measurements of gas concentration, and in the future for calibration-free operation with further high-pressure absorption model development and laser tuning characterization.
\end{abstract}

(C)2016 Optical Society of America

OCIS codes: (300.6380) Spectroscopy, modulation; (140.7260) Vertical cavity surface emitting lasers; (300.1030) Absorption; (280.1740) Combustion diagnostics; (280.4788) Optical sensing and sensors.

\section{References and links}

1. “Monthly Energy Review January 2016," U.S. Energy Information Administration.

2. R. K. Hanson, "Applications of quantitative laser sensors to kinetics, propulsion and practical energy systems," Proc. Combust. Inst. 33(1), 1-40 (2011).

3. K. Sun, X. Chao, R. Sur, J. B. Jeffries, and R. K. Hanson, "Wavelength modulation diode laser absorption spectroscopy for high-pressure gas sensing," Appl. Phys. B 110(4), 497-508 (2013).

4. M. G. Allen, "Diode laser absorption sensors for gas-dynamic and combustion flows," Meas. Sci. Technol. 9(4), 545-562 (1998).

5. J. A. Silver, "Frequency-modulation spectroscopy for trace species detection: theory and comparison among experimental methods," Appl. Opt. 31(6), 707-717 (1992).

6. G. B. Rieker, J. B. Jeffries, and R. K. Hanson, "Measurements of high-pressure $\mathrm{CO} 2$ absorption near $2.0 \mu \mathrm{m}$ and implications on tunable diode laser sensor design," Appl. Phys. B 94(1), 51-63 (2009).

7. D. S. Bomse, A. C. Stanton, and J. A. Silver, "Frequency modulation and wavelength modulation spectroscopies: comparison of experimental methods using a lead-salt diode laser," Appl. Opt. 31(6), 718-731 (1992).

8. R. Arndt, "Analytical Line Shapes for Lorentzian Signals Broadened by Modulation,” J. Appl. Phys. 36(8), 2522-2524 (1965).

9. V. Jayaraman, J. Jiang, B. Potsaid, G. Cole, J. Fujimoto, and A. Cable, "Design and performance of broadly tunable, narrow line-width, high repetition rate 1310nm VCSELs for swept source optical coherence tomography," in C. Lei and K. D. Choquette, eds. (2012), p. 82760D.

10. V. Jayaraman, G. D. Cole, M. Robertson, A. Uddin, and A. Cable, "High-sweep-rate $1310 \mathrm{~nm}$ MEMS-VCSEL with $150 \mathrm{~nm}$ continuous tuning range," Electron. Lett. 48(14), 867-869 (2012).

11. S. T. Sanders, J. A. Baldwin, T. P. Jenkins, D. S. Baer, and R. K. Hanson, "Diode-laser sensor for monitoring multiple combustion parameters in pulse detonation engines," Proc. Combust. Inst. 28(1), 587-594 (2000).

12. V. Ebert, T. Fernholz, C. Giesemann, H. Pitz, H. Teichert, J. Wolfrum, and H. Jaritz, "Simultaneous diode-laserbased in situ detection of multiple species and temperature in a gas-fired power plant," Proc. Combust. Inst. 28(1), 423-430 (2000). 
13. V. Nagali and R. K. Hanson, "Design of a diode-laser sensor to monitor water vapor in high-pressure combustion gases," Appl. Opt. 36(36), 9518-9527 (1997).

14. A. W. Caswell, S. Roy, X. An, S. T. Sanders, F. R. Schauer, and J. R. Gord, "Measurements of multiple gas parameters in a pulsed-detonation combustor using time-division-multiplexed Fourier-domain mode-locked lasers," Appl. Opt. 52(12), 2893-2904 (2013).

15. K. Kohse-Höinghaus, R. S. Barlow, M. Aldén, and J. Wolfrum, "Combustion at the focus: laser diagnostics and control," Proc. Combust. Inst. 30(1), 89-123 (2005).

16. A. W. Caswell, S. Roy, X. An, S. T. Sanders, F. R. Schauer, and J. R. Gord, "Measurements of multiple gas parameters in a pulsed-detonation combustor using time-division-multiplexed Fourier-domain mode-locked lasers," Appl. Opt. 52(12), 2893-2904 (2013).

17. T. Fernholz, H. Teichert, and V. Ebert, "Digital, phase-sensitive detection for in situ diode-laser spectroscopy under rapidly changing transmission conditions," Appl. Phys. B 75(2-3), 229-236 (2002).

18. G. Gao, B. Chen, and T. Cai, "Simultaneous detection of $\mathrm{CO}$ and $\mathrm{CO} 2$ at elevated temperatures using tunable diode laser absorption spectroscopy near $1570 \mathrm{~nm}$," Opt. Spectrosc. 114(3), 340-346 (2013).

19. G. B. Rieker, X. Liu, H. Li, J. B. Jeffries, and R. K. Hanson, "Measurements of near-IR water vapor absorption at high pressure and temperature," Appl. Phys. B 87(1), 169-178 (2007).

20. R. Sur, K. Sun, J. B. Jeffries, J. G. Socha, and R. K. Hanson, "Scanned-wavelength-modulation-spectroscopy sensor for $\mathrm{CO}, \mathrm{CO} 2, \mathrm{CH} 4$ and $\mathrm{H} 2 \mathrm{O}$ in a high-pressure engineering-scale transport-reactor coal gasifier," Fuel 150, 102-111 (2015).

21. A. Farooq, J. B. Jeffries, and R. K. Hanson, "High-pressure measurements of CO2 absorption near $2.7 \mu$ m: Line mixing and finite duration collision effects," J. Quant. Spectrosc. Radiat. Transf. 111(7-8), 949-960 (2010).

22. C. S. Goldenstein, R. M. Spearrin, J. B. Jeffries, and R. K. Hanson, "Wavelength-modulation spectroscopy near $2.5 \mu \mathrm{m}$ for $\mathrm{H} 2 \mathrm{O}$ and temperature in high-pressure and -temperature gases," Appl. Phys. B 116(3), 705-716 (2014).

23. W. R. Lempert, J. P. Looney, B. Zhang, and R. B. Miles, "Stimulated Raman scattering and coherent anti-Stokes Raman spectroscopy in high-pressure oxygen," J. Opt. Soc. Am. B 7(5), 715 (1990).

24. J. D. Miller, C. E. Dedic, S. Roy, J. R. Gord, and T. R. Meyer, "Interference-free gas-phase thermometry at elevated pressure using hybrid femtosecond/picosecond rotational coherent anti-Stokes Raman scattering," Opt. Express 20(5), 5003-5010 (2012).

25. C. E. Dedic, J. B. Michael, J. D. Miller, and T. R. Meyer, "Evaluation of Hybrid fs/ps coherent anti-Stokes Raman scattering temperature and pressure sensitivity at combustor relevant conditions," in (American Institute of Aeronautics and Astronautics, 2016).

26. D. J. Gardiner and P. R. Graves, Practical Raman Spectroscopy (Springer Science \& Business Media, 2012).

27. B. A. Stein, V. Jayaraman, J. Y. Jiang, A. Cable, and S. T. Sanders, "Doppler-limited H2O and HF absorption spectroscopy by sweeping the $1,321-1,354 \mathrm{~nm}$ range at $55 \mathrm{kHz}$ repetition rate using a single-mode MEMStunable VCSEL," Appl. Phys. B 108(4), 721-725 (2012).

28. M. Abe, S. Kusanagi, Y. Nishida, O. Tadanaga, H. Takenouchi, and H. Sasada, "Dual wavelength 3.2- $\mu$ m source for isotope ratio measurements of (13)CH(4)/(12)CH(4)," Opt. Express 23(17), 21786-21797 (2015).

29. P. Kluczynski and O. Axner, "Theoretical description based on Fourier analysis of wavelength-modulation spectrometry in terms of analytical and background signals," Appl. Opt. 38(27), 5803-5815 (1999).

30. L. C. Philippe and R. K. Hanson, "Laser diode wavelength-modulation spectroscopy for simultaneous measurement of temperature, pressure, and velocity in shock-heated oxygen flows," Appl. Opt. 32(30), 60906103 (1993).

31. G. B. Rieker, J. B. Jeffries, and R. K. Hanson, "Calibration-free wavelength-modulation spectroscopy for measurements of gas temperature and concentration in harsh environments," Appl. Opt. 48(29), 5546-5560 (2009).

32. K. Sun, X. Chao, R. Sur, C. S. Goldenstein, J. B. Jeffries, and R. K. Hanson, "Analysis of calibration-free wavelength-scanned wavelength modulation spectroscopy for practical gas sensing using tunable diode lasers," Meas. Sci. Technol. 24(12), 125203 (2013).

33. C. S. Goldenstein, C. L. Strand, I. A. Schultz, K. Sun, J. B. Jeffries, and R. K. Hanson, "Fitting of calibrationfree scanned-wavelength-modulation spectroscopy spectra for determination of gas properties and absorption lineshapes," Appl. Opt. 53(3), 356-367 (2014).

34. X. Chao, J. B. Jeffries, and R. K. Hanson, "Wavelength-modulation-spectroscopy for real-time, in situ NO detection in combustion gases with a $5.2 \mu \mathrm{m}$ quantum-cascade laser," Appl. Phys. B 106(4), 987-997 (2012).

35. "Modeling MEMS and NEMS," https://www.crcpress.com/Modeling-MEMS-and-NEMS/PeleskoBernstein/p/book/9781584883067.

36. H. Li, G. B. Rieker, X. Liu, J. B. Jeffries, and R. K. Hanson, "Extension of wavelength-modulation spectroscopy to large modulation depth for diode laser absorption measurements in high-pressure gases," Appl. Opt. 45(5), 1052-1061 (2006).

37. M. Y. Perrin and J. M. Hartmann, "Temperature-dependent measurements and modeling of absorption by CO2$\mathrm{N} 2$ mixtures in the far line-wings of the $4.3 \mu \mathrm{m} \mathrm{CO} 2$ band," J. Quant. Spectrosc. Radiat. Transf. 42(4), 311-317 (1989).

38. S. A. Clough, F. X. Kneizys, and R. W. Davies, "Line shape and the water vapor continuum," Atmos. Res. 23(34), 229-241 (1989). 
39. J. T. C. Liu, J. B. Jeffries, and R. K. Hanson, "Large-modulation-depth $2 \mathrm{f}$ spectroscopy with diode lasers for rapid temperature and species measurements in gases with blended and broadened spectra," Appl. Opt. 43(35), 6500-6509 (2004).

\section{Introduction}

Despite the rapid development of renewable energy resources, more than $80 \%$ of U.S. energy is still supplied by combustion [1]. Minimizing emissions while maximizing economy and performance of combustion devices requires diagnostic tools that are fast, sensitive, and robust against the harsh environment of the combustor. Advanced gas turbine combustors are operating at pressures $>50 \mathrm{~atm}$, commercial coal gasifiers at $>80 \mathrm{~atm}$, and liquid-fueled rocket combustors at $>100$ atm. Laser absorption diagnostics are ideal for measurements in combustion applications because they are non-intrusive, quantitative, and require only simple optical access to the combustor [2]; however, existing laser absorption techniques have not yet reached higher than 30-50 atm at room temperature. This paper presents a diagnostic technique capable of measurement at extremely high densities ( $>2.5$ times higher than previously published wavelength modulation spectroscopy measurements [3]). The technique combines the rapid, broad wavelength tuning of a Micro-Electro-Mechanical Systems (MEMS) Vertical Cavity Surface Emitting Laser (VCSEL) with the high sensitivity of Wavelength Modulation Spectroscopy (WMS).

Traditional tunable diode laser absorption spectroscopy (TDLAS) involves probing a sample region with light from a diode laser at a wavelength corresponding to a quantum transition of a gas species of interest. The amount of light absorbed as the laser passes through the region can be related back to the temperature, pressure, and concentration of the absorbing species [2,4]. Wavelength Modulation Spectroscopy (WMS) is a derivative of TDLAS that can achieve several times greater sensitivity [5]. It also has several desirable attributes at high gas densities, such as reduced sensitivity to non-ideal lineshape effects and a method for normalizing laser intensity fluctuations even when the broadening of spectral features obscures the non-absorbing baseline [6]. For the WMS technique, a fast (kHz to $\mathrm{MHz}$ ) modulation is applied to the laser to rapidly dither the wavelength across an absorption feature. The rapid tuning of the laser across the absorption feature gives rise to a series of harmonics in the detector signal at multiples of the fast modulation frequency. The magnitude of the harmonics is related to the absorption feature magnitude and shape, and can therefore be interpreted back to temperature, pressure, and absorbing species concentration as with direct absorption spectroscopy. The harmonics can be isolated with a lock-in amplifier, diminishing noise and lending sensitivity benefits to WMS $[5,7]$.

The difficulty of absorption-based measurements in high-pressure systems is demonstrated in Fig. 1. Figure 1(a) shows that for small molecules at atmospheric pressure, the absorbance spectrum is composed of distinct, narrow features corresponding to individual rotational-vibrational transitions of the absorbing gas. As gas pressure increases, so does density, leading to increased collisional effects, which broaden and blend absorption features together.

For wavelength modulation spectroscopy, the commonly used second harmonic signal (WMS-2f) is maximized when the modulation amplitude is 2.2 times the half width at half maximum (HWHM) of the absorption feature [8]. For traditional distributed feedback (DFB) diode lasers, the limit on the fast-tuning range of the laser source $(<1 \mathrm{~nm})$ limits the maximum achievable modulation amplitude, causing the WMS-2f signal to decrease as density increases and absorption features broaden past achievable tuning range of the diode. Thorlabs Inc., together with Praevium Research Inc., build medical imaging tools based on MEMS-VCSELs where broad and fast tuning is required. In our application, we employ a Praevium Research MEMS-VCSEL much like the one described in $[9,10]$, operating at $1550 \mathrm{~nm}$ and with a tuning range of $160 \mathrm{~nm}$ at modulation frequencies of hundreds of $\mathrm{kHz}$. This allows us to maintain the maximum modulation amplitude as pressure increases above $50 \mathrm{~atm}$ at room temperature (45 amagat density), as shown in Fig. 1. Figure 1(b) further illustrates this 
advantage of the wide-scanning laser. As pressure and collision broadening increase, the DFB laser is unable to maintain the optimal modulation amplitude and the peak WMS-2f signal decreases significantly for pressures above $5 \mathrm{~atm}$ (4.6 amagat at room temperature). The MEMS-VCSEL laser continues to have a strong signal because it is able to scan the optimal modulation amplitude as features broaden together. Gas density ultimately determines the level of collisional broadening, so we describe measurement conditions in terms of density to regularize between the many different combinations of pressure and temperature in past work.

Several groups have demonstrated the utility of direct laser absorption spectroscopy in high gas density environments up to 18 amagat (20 atm at $296 \mathrm{~K}$ ) [11-16]. WMS measurements have also been demonstrated at elevated density. In 2002, Fernholz et al measured $\mathrm{O}_{2}$ at pressures up to $12 \mathrm{~atm}$ (11 amagat at room temperature) [17]. Most recently, Gao et al utilized WMS to measure high pressure $\mathrm{CO}_{2}$ at densities up to 5.5 amagat (pressures of 1-10 atm at temperatures 500-1000K) [18]. Sur et al, Sun et al, Farooq et al, and Rieker et al performed WMS at densities up to 18 amagat (pressures of 20 atm at $296 \mathrm{~K}$ ) [3,6,19-21], and Goldenstein et al measured water vapor at up to 13 amagat ( $50 \mathrm{~atm}$ at $2500 \mathrm{~K}$ ) by performing scanned 2f/1f WMS using lasers around $2.5 \mu \mathrm{m}$ [22]. Despite the success of these measurements, none have pushed to densities above 18.5 amagat (equivalent to $25 \mathrm{~atm}$ at room temperature and $103 \mathrm{~atm}$ at $1500 \mathrm{~K}$ ). Other laser sensing techniques have been demonstrated at high densities, such as the work of Lempert et al measuring $\mathrm{O}_{2}$ at densities up to 31 amagat using stimulated Raman scattering and coherent anti-Stokes Raman spectroscopy (CARS) [23], and more recent CARS work in combustion [24,25]. CARS and other forms of Raman spectroscopy have been used in a laboratory setup on gases, liquids and solids past 100 amg [26].
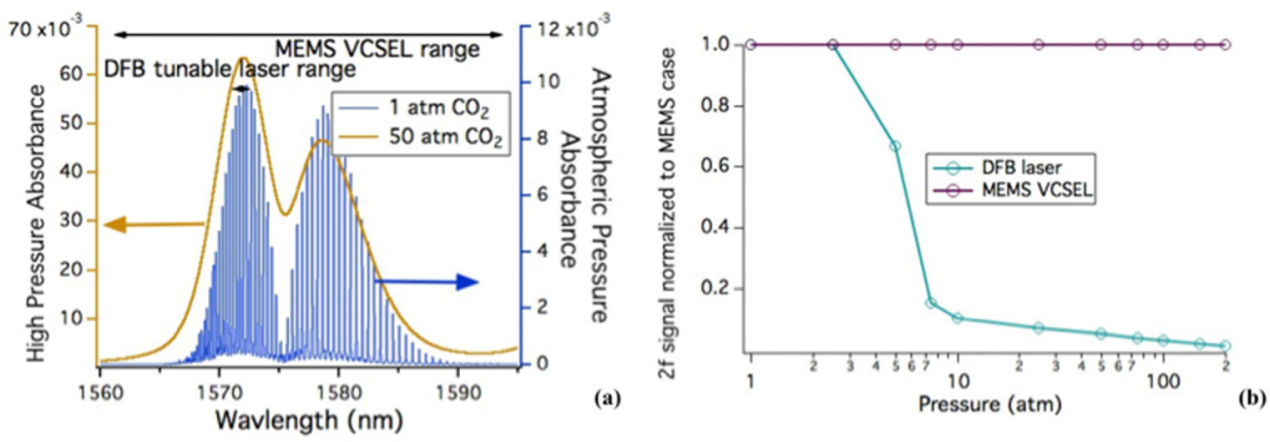

Fig. 1. (a) Room temperature absorbance spectrum of carbon dioxide $\left(\mathrm{CO}_{2}\right)$ at 1 atm and 50 atm, with the corresponding fast-scanning ranges of a traditional DFB diode laser and a MEMS-VCSEL laser (5\% $\mathrm{CO}_{2}$ in air, $100 \mathrm{~cm}$ path length). (b) Simulated $2 \mathrm{f}$ peak signal for $\mathrm{CO}_{2}$ using a DFB and the MEMS-VCSEL laser centered around $1570 \mathrm{~nm}$, normalized to the MEMS case, at a series of pressures. As pressure rises above a few atmospheres the DFB laser can no longer reach the optimal modulation amplitude for the broadening absorption conditions.

In this paper, we will describe and demonstrate the extension of WMS to the measurement of extremely high-density gases using a MEMS-VCSEL laser. Although there have been previous absorption spectroscopy experiments using a similar MEMS-VCSEL source, such as that of Stein et al using a $1310 \mathrm{~nm}$ source to perform high speed TDLAS measurements on $\mathrm{HF}$ and $\mathrm{H}_{2} \mathrm{O}$ [27], this is the first demonstration of WMS using such a source. We develop the simulations of the harmonic signals that are required to extract information from the measured data. We discuss the unique attributes of the technique, such as accounting for the nonlinear tuning of the MEMS-VCSEL laser over the large tuning range and a method to characterize the rapid, broad tuning of the laser wavelength. We demonstrate the large amplitude WMS technique on high-pressure carbon dioxide in air at densities up to 46 amagat (50 atm at room temperature). In principle the technique and laser are capable of far greater 
densities. Finally, we compare the data to simulation. The large amplitude WMS reported here can in principle be applied to any absorber exhibiting a wavelength-dependent absorption spectrum (which will induce a second harmonic signal if the laser is modulated across a significant spectral feature). Therefore, the technique may be useful for any species demonstrating broad absorption features, such as large molecular species and liquids. The technique will benefit from further evolution of the MEMS-VCSEL technology to new wavelength ranges [28].

\section{Wavelength modulation spectroscopy}

The basic theory of WMS experiments and modeling has been well described in the literature $[7,8,29,30]$; so, only the theory relevant to WMS with large modulation of MEMS-VCSELs will be discussed in this paper. WMS is performed by rapidly dithering the wavelength of a laser over an absorption feature of a species of interest. The fast modulation results in a series of harmonics on the detected laser intensity, occurring at multiples of the fast modulation frequency, $f$. In a typical DFB laser, the modulation is applied via injection current tuning, which induces temperature change and subsequent wavelength tuning, along with intensity modulation of the laser. The thermal tuning and intensity modulation under this situation is primarily linear since the tuning range is small $(<1 \mathrm{~nm})$ and any wavelength dependent loss is monotonic. The second harmonic signal, $2 f$, is the result of absorption, because the laser wavelength is tuned back and forth across the absorption feature during each cycle of the modulation. Thus, the $2 f$ signal (and higher even harmonics) is of particular interest because it is sensitive to absorption parameters such as the strength and the shape of the feature, which can enable the extraction of thermodynamic properties of the gas. The harmonic signals are individually isolated using a lock-in amplifier.

In order to extract the thermodynamic gas properties from a WMS measurement the measured harmonic signal from the lock-in is compared with a simulation of the expected signal that includes both the absorption properties of the target species and the laser modulation properties [31]. The WMS simulation must therefore account for the unique intensity and wavelength tuning qualities of the MEMS-VCSEL. In the following section, we present a technique for incorporating these unique qualities of the MEMS-VCSEL laser into WMS simulations.

\section{Impact of laser tuning characteristics on WMS with a MEMS VCSEL}

Traditionally, WMS harmonic signals are simulated using Fourier expansion of the spectral absorbance [7,30,31]. However, the Fourier expansion becomes intractable when the laser intensity and wavelength modulation are highly nonlinear. Unlike typical injection current tuning, the MEMS-VCSEL changes the laser cavity length using electrostatic actuation. Electrostatic tuning does not directly change the intensity of the laser. Instead, the wavelength-dependent features of the laser mirrors, gain medium, and experimental system components (transmit/receive optics, optical cell windows, etc.) cause intensity modulation as the wavelength is tuned. Over the wide tuning range of the MEMS-VCSEL $(\sim 160 \mathrm{~nm})$, these features are not monotonic and therefore create higher order frequencies in the intensity. The MEMS-VCSEL's nonlinear intensity profile during wavelength scanning negates the linearity assumptions often applied to DFB laser-based WMS, where a Fourier expansion is employed. Instead, we use a new approach similar to Refs [32-34] where we directly model the expected transmitted intensity of the laser through the sample region using the actual measured incident intensity and Beer's Law, Eq. (1).

$$
I_{t}(t)=I_{0}(t) e^{\alpha(\lambda(t)))}
$$

where $I_{0}$ is the incident intensity, $\alpha$ is absorbance, and $\lambda(t)$ is the wavelength as a function of time. We then process the simulated transmitted intensity with a digital lock-in amplifier that isolates the harmonic signal of interest. 
This technique requires three inputs: (1) $\mathrm{I}_{0}(\mathrm{t})$, the incident laser intensity (before absorption), (2) $\lambda(t)$, wavelength as a function of time, and (3) $\alpha(\lambda)$, absorbance as a function of wavelength. Each input will be explained in detail in the following subsections.

\subsection{Incident intensity characterization, $I_{0}$}

To simulate the transmitted intensity, $\mathrm{I}_{\mathrm{t}}(\mathrm{t})$, via Beer's law we need to first measure the intensity incident on the gas sample, which is denoted $\mathrm{I}_{0}$. The MEMS-VCSEL laser used for this experiment is capable of scanning $\sim 160 \mathrm{~nm}$ centered around $1570 \mathrm{~nm}$ at frequencies up to $400 \mathrm{kHz}$. A drive voltage is passed to the laser, which electrostatically pulls down the MEMS mirror that forms the optical cavity above the VCSEL. The laser exhibits nonlinear relationships between the drive signal and both laser intensity and wavelength. Figure 2 shows the laser intensity variation when a fast sinusoidal modulation signal is passed to the laser. Figure 2(b) illustrates the non-sinusoidal intensity signal that results from the application of this fast sinusoidal modulation (Fig. 2(a)). Figure 2(d) shows the laser intensity when a lower frequency linear voltage sweep (Fig. 2(c)) is also applied to slowly tune the center wavelength about $25 \mathrm{~nm}$ across the absorption features (as required to obtain a wavelength-resolved WMS spectrum). It is evident that the amplitude of the laser intensity modulation is center-wavelength-dependent. Therefore, the laser intensity cannot be simulated by a simple sinusoid, as is done in traditional WMS modeling. Instead, we measure the laser output intensity directly and incorporate the measured output intensity variation into the simulation.
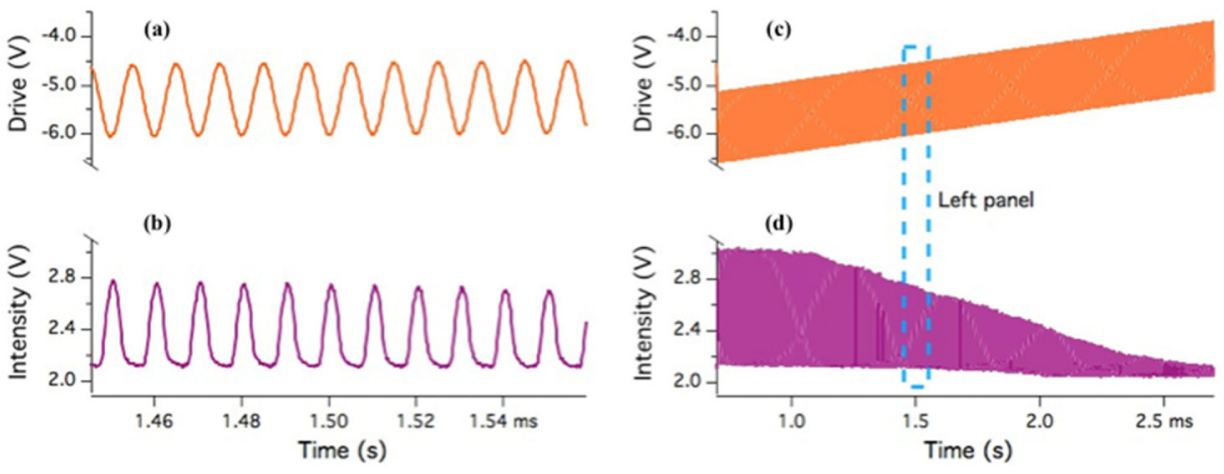

Fig. 2. (a) Sinusoidal voltage input signal to the laser used to modulate the laser wavelength (100 kHz modulation frequency); (b) Resulting intensity variation as a function of time, measured concurrently with the voltage input; (c) Voltage input signal as center wavelength is tuned with additional $500 \mathrm{~Hz}$ voltage sweep; (d) Resulting intensity variation for one full voltage sweep with fast modulation applied.

In order to track laser output, we need to measure both the temporal variation of the laser intensity and its distortion due to non-linear system effects. These characterizations require two separate measurements.

To account for temporal variation, we make a concurrent, reference intensity measurement of the light coming from the laser. This is achieved by passing the laser light through a fiber splitter and sending half of the light to a photodetector, allowing for a concurrent intensity measurement of laser output, denoted as $I_{r e f}$. This characterization setup is shown in the experimental set up in Sec. 4.

To account for distortion due to elements of the optical setup (cell windows, etc.), we characterize the system response by passing a laser through the measurement cell when there is only non-absorbing gas present. This measurement accounts for etalons and optical effects particular to the experimental setup, such as variations in light transmission due to pressure- 
induced stress on the windows. This measurement is denoted $I_{n a}$ to denote non-absorbing background.

We combine these measurements in Eq. (2) by taking the ratio of the reference intensity during the sample measurement ( $\mathrm{I}_{\text {ref,sample }}$ ) to the reference measurement taken concurrently with the non-absorbing measurement through the cell $\left(\mathrm{I}_{\text {ref,na }}\right)$, which is then multiplied by the transmitted intensity through the non-absorbing gas in the cell $\left(\mathrm{I}_{n a}\right)$.

$$
\mathrm{I}_{0}=\left(\frac{\mathrm{I}_{\text {ref,sample }}}{\mathrm{I}_{\text {ref,na }}}\right) \mathrm{I}_{n a}
$$

The resulting $\mathrm{I}_{0}$ represents the incident intensity that we would expect to measure for the system of interest, corrected for both the non-uniformities in the optical cell and temporal drifts of the laser itself.

\subsection{Wavelength characterization, $\lambda(t)$}

It is understood that electrostatic MEMS mirrors reduce the cavity length of the laser as the square of the applied voltage [35] We expect, as with intensity variation, the wavelength of the MEMS laser will not vary linearly with the drive modulation, so the standard WMS model assumption of a constant sinusoidal wavelength modulation is not valid.

The combination of the extremely large wavelength sweep range and fast sweep frequency of the laser makes large amplitude WMS possible, but also makes wavelength characterization difficult. Typically the wavelength variation of a laser can be characterized using a combination of an optical spectrum analyzer (OSA), a wavemeter, and/or an etalon with a well-known free spectral range [36]. For typical DFB lasers, the fast and slow modulations can be considered independent and can thus be turned off to perform a two-part characterization. First, an absolute characterization of the center wavelength with the modulation turned off is performed with an OSA or wavemeter (which have slow acquisition rates). Then, the modulation is turned on and an etalon is used to provide a relative measurement of the wavelength modulation amplitude and slow scanning of the center wavelength (if used). Because the modulation amplitude varies significantly as the center wavelength of the MEMS-VCSEL is scanned, this characterization procedure does not work well for the MEMS-VCSEL.

Instead, we use a reference cell containing Carbon Monoxide together with a high-speed data acquisition system to provide absolute wavelength characterization at high speed. A NIST SRM 2515 Carbon Monoxide Absorption Reference Cell consists of a small chamber of ${ }^{12} \mathrm{C}^{16} \mathrm{O}$ kept at a well-known temperature and pressure, such that the line-center wavelength of each $\mathrm{CO}$ absorption line is well known. The $\mathrm{CO}$ absorption cell thus provides an absolute wavelength reference as the laser sweeps over the known $\mathrm{CO}$ absorption lines, even during a fast scan. An example of the characterization using this method is shown in Fig. 3, where Fig. 3 (a) shows the transmitted intensity of the laser through the reference cell during a single cycle of the fast modulation and the Fig. 3(b) shows the conversion to wavelength as a function of time based on the $\mathrm{CO}$ peak locations. The conversion from intensity measurement to wavelength is done by applying a peak finding algorithm to the transmitted intensity spectrum and matching the corresponding peaks to the known absolute center wavelengths of the ${ }^{12} \mathrm{C}^{16} \mathrm{O}$ absorption features. We repeat the process to characterize fast modulation cycles at multiple locations throughout the slow voltage sweep. 

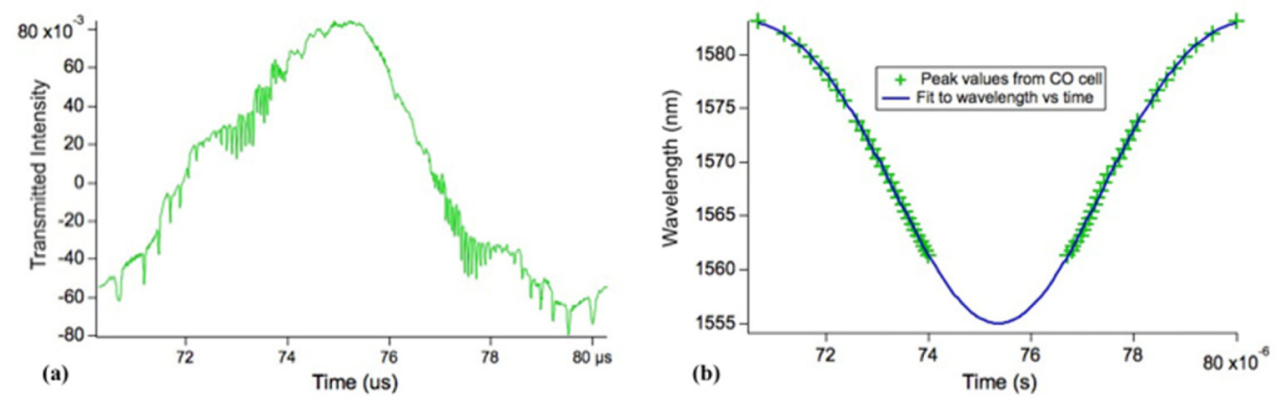

Fig. 3. (a) Transmitted intensity through the SRM $2515 \mathrm{CO}$ cell as a function of time when the fast sine wave voltage modulation is applied to the laser. Variations in the baseline laser intensity through the $\mathrm{CO}$ cell are due in part to the nonlinear laser intensity modulation of the source and etalon effects in the un-optimized reference cell; (b) Wavelength as a function of time determined from the data in the left panel: location of $\mathrm{CO}$ absorption peaks (points) and curve fit (line).

Each characterization results in a center wavelength, and first and second harmonic modulation amplitude at a different point in the slow voltage sweep. The nature of the electrostatic modulation results in wavelength modulation that varies quadratically throughout the slow scan, so the measured wavelength variation across the slow scan is fit with Eq. (3)(resulting scan is shown in Fig. 4):

$$
\lambda(t)=\left(\lambda_{0}+\lambda_{1} t+\lambda_{2} t^{2}\right)+\left(a_{0}+a_{1} t+a_{2} t^{2}\right) \sin \left(2 \pi f t+\phi_{1}\right)+\left(b_{0}+b_{1} t+b_{2} t^{2}\right) \sin \left(4 \pi f t+\phi_{2}\right)
$$

where $\lambda_{i}$ are the terms of the second order variation of the center wavelength, $a_{i}$ are the terms of the first harmonic amplitude variation, $b_{i}$ are the terms of the second harmonic amplitude, $f$ is the frequency of the fast modulation, and $\phi_{1}$ and $\phi_{2}$ are the phase offsets between intensity and wavelength variation for the two harmonics, respectively. In future applications we will utilize a synthesized voltage waveform to account for the nonlinear voltage and wavelength relationship, as opposed to the simple sinusoid and sawtooth driving voltage used in this experiment [9].
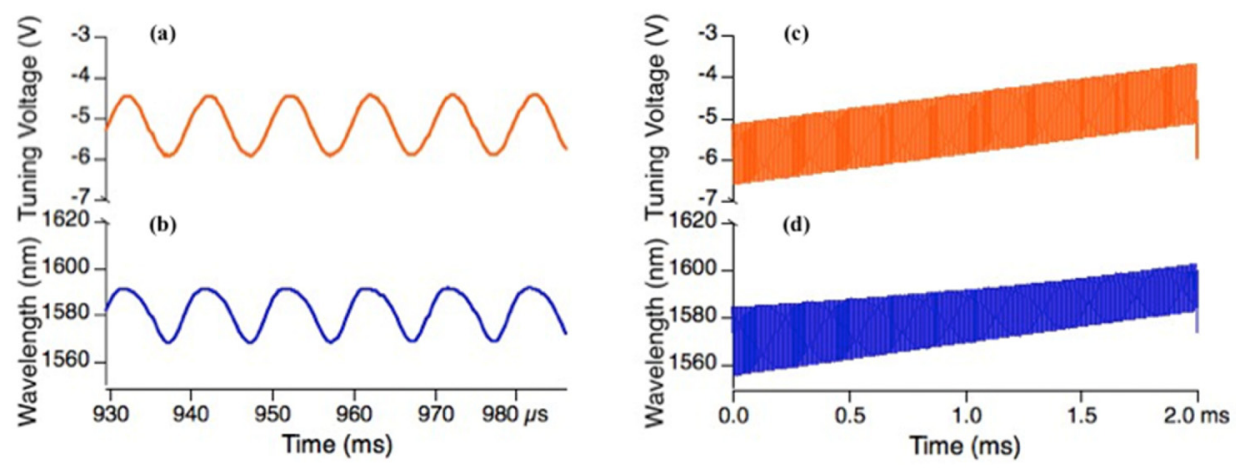

Fig. 4. (a) Sinusoidal voltage input signal to the laser used to modulate the laser wavelength (100 kHz modulation frequency); (b) Resulting wavelength variation as a function of time; (c) Voltage input signal as center wavelength is tuned with additional $500 \mathrm{~Hz}$ voltage sweep; (d) Resulting wavelength variation for one full voltage sweep with fast modulation applied.

During the characterization, up to twenty ${ }^{12} \mathrm{C}^{16} \mathrm{O}$ absorption peaks are swept twice per $\sim 10$ microsecond modulation cycle. A fast data acquisition system (NI PXIe-7975R with 5761 digitizer sampling at $250 \mathrm{MHz}$ ) is required to resolve the narrow low-pressure $\mathrm{CO}$ absorption lines in the reference cell. The input to the fast DAQ is low impedance and the laser tuning 
input is high impedance. The low impedance DAQ alters the laser tuning voltage if the drive voltage is simultaneously connected to the laser and the fast DAQ. Therefore to avoid alteration of the drive signal during characterization, we did not measure the laser input voltage simultaneously during wavelength characterization. Because neither the concurrent intensity nor the laser tuning voltage are measured concurrently with the wavelength characterization, the phase shifts between the laser intensity and laser wavelength modulation, $\phi_{1}$ and $\phi_{2}$, are not directly measured for the experiments in this paper (shown in Fig. 4): Therefore, we had to infer these phase shifts during the comparison of the model to the experiments. Future implementations will simultaneously measure laser intensity on the fast DAQ during wavelength characterization to measure these phase shifts.

\subsection{Absorbance model, $\alpha(\lambda)$}

We utilized the spectral database HITRAN 2012 together with Voigt absorption profiles to simulate the spectral absorbance, $\alpha(\lambda)$, as shown in Fig. 1(a). This method is known to be inaccurate for simulating high pressure absorbance due to the breakdown of the assumptions inherent to the Lorentzian collisional broadening model used in the Voigt profile, in addition to line mixing, and other effects [6]. However, improved data on $\mathrm{CO}_{2}$ broadening at high densities does not yet exist to provide an improved model. Fortunately, it has been shown that the impact of these non-ideal effects are lessened for WMS (vs. direct absorption spectroscopy) [6]. Still, we expect error in the simulated WMS signal induced by inaccuracy of the absorbance model at high-density conditions.

To assess the impact of the error in the absorbance model, the comparisons in Sec. 4 show an additional WMS simulation that includes a wavelength-dependent scaling to account for the breakdown of the Voigt profile at high $\mathrm{CO}_{2}$ densities. Several empirical corrections have been developed to account for the shift of absorbance from the wings of an absorption feature toward the center due to non-Lorentzian effects at high densities. We utilized the wavelengthdependent empirical correction developed by Perrin and Hartman, which is multiplied by the simulated absorbance for each individual spectral line [37]. The model defined by Perrin and Hartman accounts for the reduction of absorption in the far wings of features, but does not conserve the integrated absorbance by enhancing absorption near line center (as expected, and shown in similar corrections developed for water vapor [38]). We therefore enhanced the scaling near the center region of the piece-wise $\chi$-function correction to conserve the integrated absorbance for each absorption line. We emphasize that this correction was applied to determine the influence of the breakdown of the Voigt profile on the WMS simulation, and is not an alternative to future development of proper high-density absorption models. Accounting for linemixing and other effects is also necessary to further improve the fidelity of the absorption models [21].

\section{Demonstration}

To test the MEMS-VCSEL large amplitude WMS technique, we perform measurements on samples of high-density room temperature $\mathrm{CO}_{2}$ (equivalent density to $255 \mathrm{~atm}$ at $1500 \mathrm{~K}$ ). Note that the technique is capable of probing even higher densities or broader spectra, as these experiments only use a modulation amplitude of $\sim 30 \mathrm{~nm}$, while the laser is able to modulate up to $160 \mathrm{~nm}$. We selected carbon dioxide because it is a major product of combustion and has absorption features in the wavelength range of the MEMS-VCSEL laser that are relatively free from water vapor absorption interference. For the large amplitude WMS technique to be useful as a gas concentration sensor, we must be able to interpret the measured signals with a simulation. Therefore the goal of the experiment was to demonstrate that the measured large-amplitude WMS data at high density match simulation and scale linearly with concentration. 
A schematic of the experimental setup is shown in Fig. 5, including the wavelength and intensity characterization setups. The wavelength characterization does not need to be performed synchronously with the sample measurement.

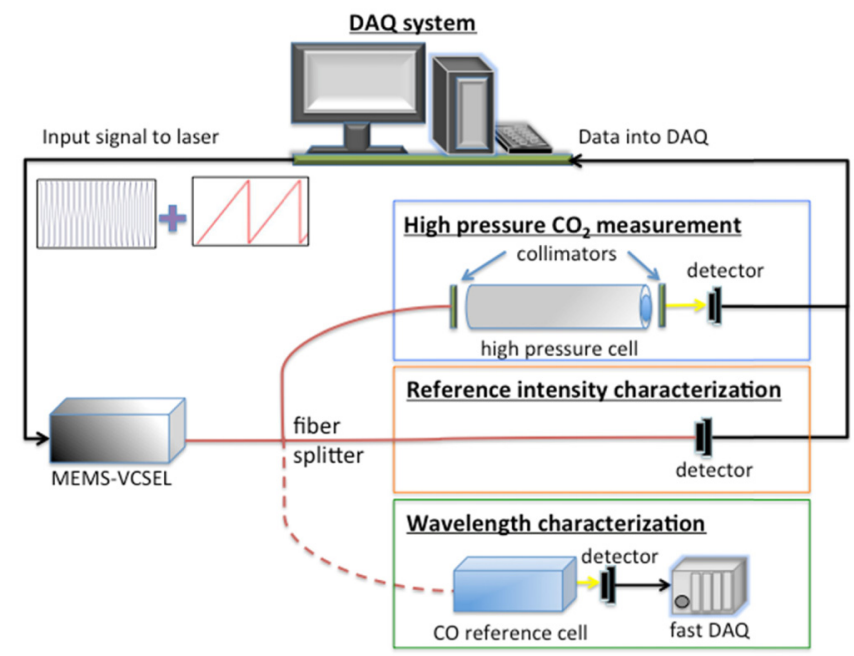

Fig. 5. Experimental setup for large amplitude WMS of high pressure $\mathrm{CO}_{2}$ using a MEMSVCSEL. The laser intensity is characterized simultaneously with the high pressure cell measurements, and the wavelength is characterized before or after the experiment. The system listed as the DAQ system is a desktop computer with a NI PCI 6110 while the fast DAQ is the NI PXIe-7975R.

A National Instruments PCI 6110 data acquisition board outputs the drive voltage to the laser and records the transmitted intensity through the $\mathrm{CO}_{2}$ cell and directly to the intensity characterization detector. The laser drive signal, combines a $500 \mathrm{~Hz}$ sawtooth sweep of the center wavelength and a $100 \mathrm{kHz}$ fast sinusoidal modulation. The drive signal is also recorded to the DAQ system as a reference to synchronize simulated $\mathrm{I}_{0}$ signals with the measured data. The DAQ system samples at $5 \mathrm{MHz}$, which is more than sufficient to capture the second harmonic detector signal at $200 \mathrm{kHz}$.

A software lock-in amplifier isolates the WMS second harmonic from the transmitted intensity signal. To be consistent, the same software lock-in amplifier is used to separately post-process both the measured data and the simulated transmitted intensity that forms the basis of the WMS model.
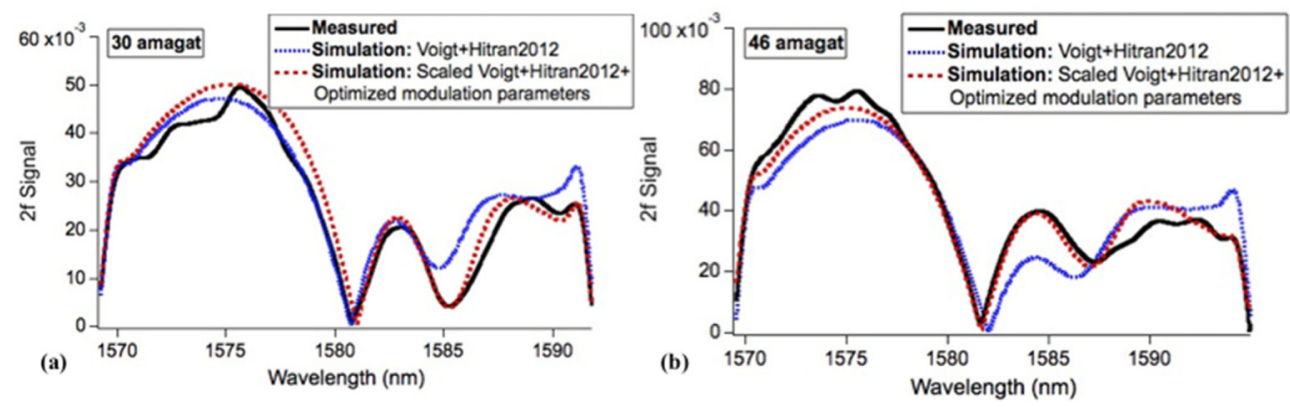

Fig. 6. (a) Simulated and measured second harmonic signal for $14.7 \% \mathrm{CO}_{2}$ mixed with air at 30 amagat (32.7 atm at 295K). (b) Simulated and measured second harmonic signal for $12 \%$ $\mathrm{CO}_{2}$ mixed with air at a density of 46 amagat $(50.5 \mathrm{~atm}$ at $300 \mathrm{~K})$. The red dash trace was obtained by scaling the simulated Voigt profile with a modified $\chi$-function correction to account for non-Lorentzian lineshape effects that occur at high density, and by optimizing the wavelength modulation parameters within their characterization uncertainty. 
The highly nonlinear intensity modulation of the laser signal induces a strong residual amplitude modulation (RAM) background signal - i.e. there is a large (but stable) background WMS second harmonic signal even when an absorbing gas is not present. This is typical (though at a smaller magnitude) even in diode laser-based WMS, and the signal is normally subtracted from both the simulation and data [31]. Here, we measure the RAM background from the laser intensity characterization signal $\left(\mathrm{I}_{0}\right)$, and subtract from both the data and simulation. It is also possible to manipulate the laser output to minimize the measured background harmonic signals [9]. To perform the background subtraction we pass the simulated transmitted intensity, the measured transmitted intensity, and the $\mathrm{I}_{0}$ signal through the digital lock-in. Because the $2 f$ signal is directly proportional to laser intensity, differences in baseline intensity values among the signals will cause the absolute value of the $2 f$ magnitudes to be different. Therefore the average magnitude of each intensity signal (simulated or measured) is scaled to match before they are passed through the lock-in. The magnitude of the background-subtracted signals is then calculated from Eq. (4):

$$
2 f=\sqrt{\left(2 f_{x, \text { signal }}-2 f_{x, \text { background }}\right)^{2}+\left(2 f_{y, \text { signal }}-2 f_{x, \text { background }}\right)^{2}}
$$

Where the $\mathrm{x}$ and $\mathrm{y}$ components of the signal are the two orthogonal outputs from the lock-in [31].

In Fig. 6 we plot the measured and simulated results at 32.7 atm and $50.5 \mathrm{~atm}$. Each plot contains two simulations: one that utilizes the measured wavelength modulation parameters and standard Voigt profile absorbance model, and another that utilizes modulation parameters that are optimized within the uncertainty of the measured modulation parameters and an absorbance profile using the scaled $\chi$ function correction. The original simulation using only measured parameters and a standard Voigt profile-based model, shows general agreement with the measurement, but suffers from the known inaccuracy at high densities of the absorption model and uncertainty in the modulation parameters stemming from the difficult wavelength characterization. The second simulation explores the influence of these potential sources of uncertainty by adding the scaled $\chi$-function correction to the absorption model, and optimizing the wavelength modulation parameters by varying the $\lambda_{i}, a_{i}$, and $b_{i}$ parameters within one standard deviation of their fit values. The revised simulation, shown in dashed red, tracks more closely with the measured $2 f$ data, suggesting that these sources of uncertainty are important. Multiple reflections in the cell windows or other noise in the optical cell may account for the remaining discrepancy, particularly the ripple in the measured signal. Overall, the match between the simulation and measurement demonstrate that with refinement, the technique can be used to extract thermodynamic properties from the data without calibration.
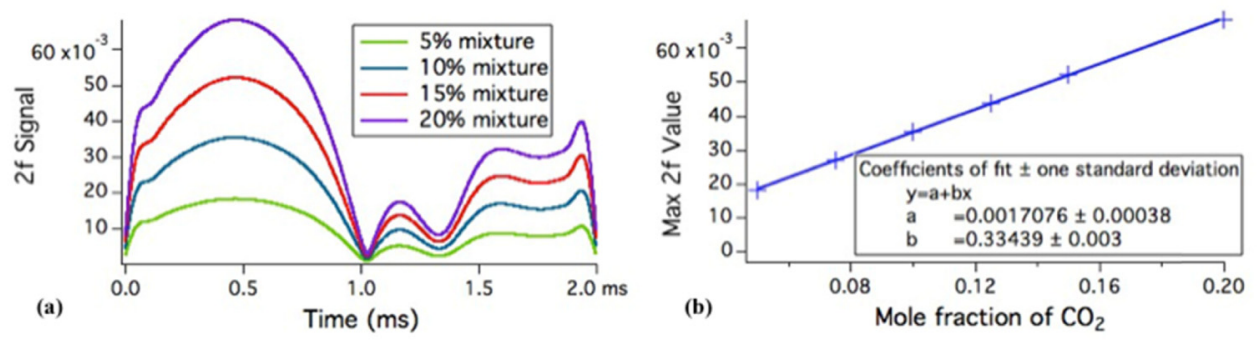

Fig. 7. (a) Simulated second harmonic signal for four different $\mathrm{CO} 2$ mole fractions in air at room temperature, $101 \mathrm{~cm}$ path length and $32.7 \mathrm{~atm}$. (b) The maximum $2 \mathrm{f}$ value from the graph on the left as a function of mole fraction.

If the measured $2 \mathrm{f}$ signal is linearly proportional to concentration, the measured signal can be used to extract concentration even without an accurate model, if a single point calibration at a known condition is possible (as was used for early demonstrations of the WMS technique 
[39]). Figure 7 shows simulations for several different concentrations. The left panel shows that the WMS-2f signal increases with the concentration of the mixture. The right panel demonstrates that the WMS-2f peak signal exhibits the expected linear relationship with species mole fraction.

\section{Conclusion}

This paper presents the extension of the sensitive wavelength modulation spectroscopy technique to large modulation depths by using a MEMS-VCSEL laser. We demonstrated the potential of the technique by performing measurements at $2.5 \mathrm{x}$ higher density than previous measurements (46.3 amagat, $50.5 \mathrm{~atm}$ at room temperature). Through extensive characterization of the laser tuning properties and implementation of a new WMS model, we demonstrate good agreement between the model and the measured data. Although discrepancies remain, the agreement suggests that this sensor has the potential to measure the thermodynamic properties such as concentration and temperature of broadly absorbing species.

\section{Acknowledgments}

The authors thank Vijaysekhar Jayaraman, Christopher Burgner, and Demis D. John of Praevium Research Inc. for helpful technical discussions. We thank Praevium Research Inc. for loaning the MEMS-VCSEL swept laser source used in this work and Thorlabs, Inc. for funding development of the laser. 